\title{
26397 - MULTIMEDIA INSTRUCTION FOR TEACHING CRISIS RESOURCE MANAGEMENT
}

\section{Timothy Welke MD, Viren Naik, MD; Georges Savoldelli, MD; Hwan Joo, Vicki LeBlanc, PhD; St. Michael's Hospital, University Of Toronto, Toronto, ONTARIO, Canada}

INTRODUCTION: Several advantages of simulation over didactic and patient dependent teaching have lead to a proliferation of patient simulators in academic centers. Although debriefing following simulation is a critical component of the learning process, optimal methods of debriefing have not been well studied. Standardized multimedia instruction is useful in teaching surgical skills, but has not been evaluated for use as an adjunct in crisis management training.(1) The primary purpose of this study was to determine whether standardized computer-based multimedia instruction is effective for learning nontechnical (behavioural) skills in patient crisis scenarios. The secondary purpose was to compare multimedia debriefing to individual oral debriefing with an expert.

METHODS: After institutional ethics approval, 30 junior (postgraduate years 1 and 2) anesthesia residents were recruited to manage three different simulated resuscitation crises using a high-fidelity patient simulator. Following the first scenario, subjects were randomized to either computer-based multimedia instruction or an individual oral debriefing with an expert. Subjects then participated in an immediate posttest scenario, followed by a retention test five weeks later. The non-technical performances were independently rated by two expert and blinded assessors using the previously validated Anesthesia Non Technical Skills (ANTS) marking system.(2) Data were analyzed using an ANOVA and Tukey's post hoc comparison tests.

RESULTS: Resident performance of non-technical skills improved significantly after multimedia instruction $(\mathrm{p}<0.05)$. This improvement was sustained after five weeks $(p<0.05)$. There were no significant differences in the performance of residents receiving multimedia instruction compared to oral debriefing in any of the tests.

DISCUSSION: Computer-based multimedia instruction is an effective method of teaching non-technical skills in simulated crisis scenarios and may be as effective as a personalized oral debriefing. Multimedia instruction may be a valuable adjunct to centers when debriefing expertise is limited. Multimedia instruction may also improve simulation utilization by reducing anxiety of peer evaluation in continuing medical education.(3)

REFERENCES: (1) Surgery 1999; 126: 330-6; (2) Br J Anaesth 2003; 905 580-588 ; (3) Can J Anesth 2005

This study was supported by the physicians of Ontario through a grant by the Physicians' Services Incorporated Foundation. 




